\title{
Análisis Computacional de una Turbina Hidrocinética de Eje Horizontal con y sin Difusor
}

\section{Computational Analysis of Horizontal Axis Hydrokinetic Turbine with and without Diffuser}

\author{
C. Cardona-Mancilla ${ }^{1}$, J. A. Casas Monroy², J. Sierra Del Rio², \\ E. L. Chica Arrieta ${ }^{3}$ y D. Hincapié-Zuluaga ${ }^{2}$
}

Recibido: 15 de enero 2017

Aceptado: 18 de mayo 2017

\section{Resumen}

Las turbinas hidrocinéticas utilizan la energía contenida en el flujo de agua de mares, ríos, canales, entre otros, para generar energía eléctrica. La principal ventaja de estas turbinas es que no necesitan represas debido a que su funcionamiento es independiente de caídas o cabezas de agua, convirtiéndolas en una tecnología de bajo costo. Actualmente, los casos más exitosos de turbinas hidrocinéticas operativas se encuentran en Europa, no obstante Brasil viene marcando una gran tendencia en el estudio e implementación de las mismas. Este trabajo presenta el análisis computacional en modo transitorio de una turbina hidrocinética de eje horizontal realizado en el programa ANSYS CFX V16.2®. La turbina está constituida por tres álabes con perfil hidrodinámico NREL 5822 y ángulo de ataque de $5^{\circ}$. También es analizado un difusor, generado a partir del mismo perfil hidrodinámico, con el fin de evaluar su efecto en el comportamiento de la turbina. Una velocidad de la corriente de agua de $1.5 \mathrm{~m} / \mathrm{s}$ y una

1 Ingeniero Mecatrónico, Estudiante maestría en gestión energética industrial, Instituto Tecnológico Metropolitano - ITM, Medellín Colombia

2 Magister, Departamento de Ingeniería Mecatrónica, Facultad de Ingenierías, Instituto Tecnológico Metropolitano - ITM, Medellín Colombia

3 Doctor, Departamento de Ingeniería Mecánica, Facultad de Ingeniería, Universidad de Antioquia UdeA, Medellín, Colombia

E-mail: diegohincapie@itm.edu.co; cristiancardona@itm.edu.co 
variación de la velocidad angular entre o y 300 RPM fueron utilizadas como condiciones de operación para la simulación. Como resultado se obtiene la potencia generada en función de la velocidad angular para ambos modelos. La mayor potencia generada por la turbina con y sin difusor fue de $879.7 \mathrm{~W}$ a 180 RPM y $845.9 \mathrm{~W}$ a 200 RPM, respectivamente, equivalente a un incremento del $3.84 \%$.

Palabras clave: CFD, energía cinética, energía renovable, picogeneración eléctrica.

\section{Abstract}

Hydraulic turbines use the energy contained in the water flow of mares, rivers, canals, among others, to generate electricity. The main advantage of these turbines is that they do not need dams, due to their operation is independent of water heads, it became a low-cost technology. Currently, the most successful cases of operational hydrokinetic turbines are in Europe, but Brazil has marked a great trend in the study and implementation of these. This work presents the transient computational analysis of a horizontal axis hydrodynamic turbine made in the ANSYS CFX V $16.2^{\circledR}$ program. The turbine is constituted by three blades with hydrodynamic profile NREL S822 and angle of attack of $5^{\circ}$. It is also analyzed a diffuser, generated from the same hydrodynamic profile, to evaluate its effect on the behavior of the turbine. A water velocity of $1.5 \mathrm{~m} / \mathrm{s}$ and a variation of the angular velocity between 0 and 300 RPM were used as operating conditions for the simulation. As a result, the power generated as a function of the angular velocity is obtained for both models. The highest power generated by the turbine with and without diffuser was $879.7 \mathrm{~W}$ at $180 \mathrm{RPM}$ and $845.9 \mathrm{~W}$ at $200 \mathrm{RPM}$, respectively, equivalent to an increase of $3.84 \%$.

Keywords: CFD, kinetic energy, peak power generation, renewable energy.

\section{Introducción}

In tema de gran interés en la actualidad es la generación de energía eléctrica a partir de fuentes renovables, esto es debido a que este tipo de aplicaciones contribuye con la preservación del medio ambiente, al reducir la utilización de combustibles fósiles. Actualmente se vienen realizando investigaciones sobre la implementación de turbinas hidrocinéticas (THC) para generar energía eléctrica a partir de la extracción de la energía contenida en corrientes o flujos de fuentes hídricas, enfocadas en la viabilidad y ubicación de las mismas ${ }^{[1]}$. Se destaca la posibilidad de emplear este tipo de tecnología en diferentes localidades del mundo donde se presentan corrientes de agua a bajas velocidades con picos medios de aproximadamente $2 \mathrm{~m} / \mathrm{s}^{[2]}$.

Las THC están basadas en parámetros de diseño y operación de turbinas eólicas, pese a lo cual, existe un amplio margen con relación a la cantidad de 
información concebida sobre cada una de éstas, dejando un vacío importante por cubrir representado especialmente en los diversos perfiles de velocidades que no han sido analizados. Por tal razón, se hace necesaria la implementación de herramientas de simulación validadas que permitan el diseño y análisis de dichas turbinas, evitando así los altos costos que trae consigo la fabricación a ensayo y error ${ }^{[3]}$.

Para mejorar la potencia generada por THC de eje horizontal se ha venido fomentando el uso de difusores. Su función principal es incrementar el flujo a la entrada de la turbina y a su vez acelerarlo aguas abajo de la misma, mejorando su circulación al disminuir el estancamiento del fluido en dicha zona ${ }^{[4]}$. El límite de Betz establece que el máximo coeficiente de potencia teórico que puede extraer una THC de una corriente de agua es $C_{p, \text { máx. }}=0.593$. Por esta razón, la implementación de difusores ha tenido gran acogida en la adaptación a este tipo de turbinas, debido a que permite incrementar su coeficiente de potencia, al punto de exceder el límite de Betz ${ }^{[4]-[6]}$.

Con el fin de compensar la baja densidad de potencia generada por una turbina hidrocinética, Gaden y Bibeau, (2010) ${ }^{[6]}$, realizan un estudio numérico analizando el rendimiento y la viabilidad que representaría el uso de difusores en turbinas hidrocinéticas de flujo axial, obteniendo como resultado que al emplear un difusor con las dimensiones propuestas en su trabajo se presenta 3.1 veces más de potencia en comparación con la turbina sin dicho componente. Un estudio semejante es llevado a cabo por Shahsavarifard et al., (2013) [7], en el cual se evalúan experimentalmente tres modelos, siendo el primero de ellos una turbina hidrocinética de eje horizontal, el segundo la implementación de una cubierta exterior sobre la turbina y el tercero la misma turbina con un difusor, encontrando como resultado un incremento del $91 \%$ en la potencia máxima de salida del tercer modelo con respecto al primero.

El objetivo de este trabajo es evaluar el rendimiento hidrodinámico de un difusor en función de la generación de potencia de una turbina hidrocinética de eje horizontal. Utilizando para la elaboración geométrica del mismo el perfil hidrodinámico NREL S822, a fin de aprovechar su curvatura como un difusor convergente-divergente.

Se espera obtener un incremento en la potencia de salida de la turbina, dado los resultados presentados en la literatura al emplear este tipo de dispositivo en turbinas hidrocinéticas de eje horizontal. 


\section{Metodología}

\subsection{Elaboración del modelo}

El modelo tridimensional de la turbina hidrocinética de eje horizontal fue realizado utilizando el programa NX $10.0{ }^{\circledR}$ de SIEMENS ${ }^{\circledR}$. El rotor de $1.5 \mathrm{~m}$ de diámetro está diseñado para generar una potencia de $1 \mathrm{HP}(745.7 \mathrm{~W})$ a velocidades promedio de $1.5 \mathrm{~m} / \mathrm{s}$. La turbina está conformada por un cubo y tres álabes (Fig. 1), utilizando para estos un perfil hidrodinámico NREL S822 y un ángulo de ataque de $5^{\circ}$. Este perfil fue utilizado debido a presenta una característica de auto-limpieza, que le proporciona una mayor resistencia a la suciedad que los perfiles NACA convencionales ${ }^{[8]}$. Esta característica es relevante, debido a que pueden generarse alteraciones en el funcionamiento de la turbina causadas por basuras o sedimentos contenidos en la fuente hídrica utilizada. Para el diseño el difusor, se utilizó el mismo perfil de los álabes escalado a una proporción de 3:1, empleando para ello la sección del perfil de la base del álabe, por ser la de mayor tamaño. Esto debido a que el álabe disminuye su tamaño desde la base hasta la punta. Algunas de las dimensiones empleadas para el difusor se presentan en la Fig. 2. Para modelar el difusor se empleó el programa Solid Edge ST ${ }^{\circledR}$ de Siemens ${ }^{\circledR}$, en el cual también se llevó a cabo el respectivo ensamble con la turbina hidrocinética, teniendo en cuenta una ubicación central de la misma a lo largo y ancho del interior del difusor.

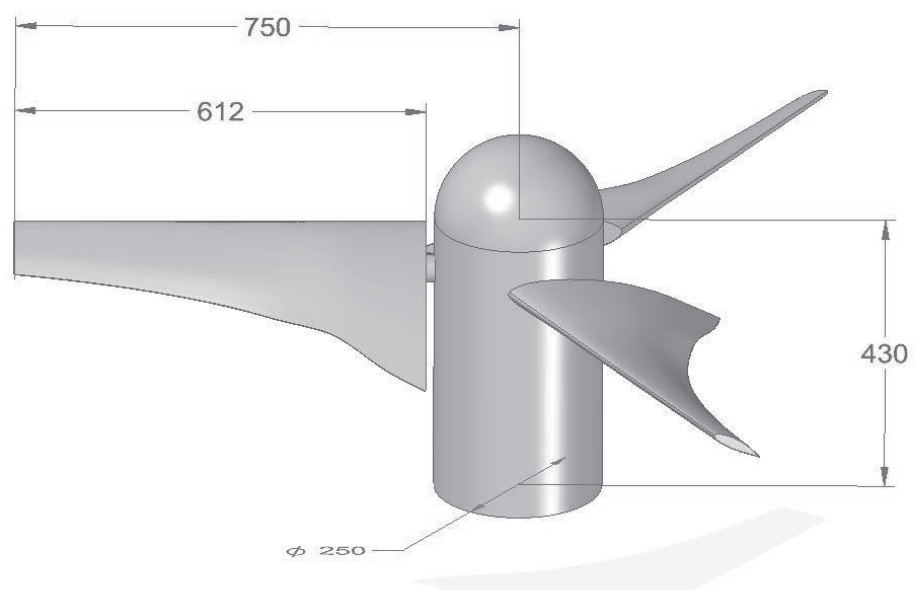

FIGURA 1. MODELO TRIDIMENSIONAL DE LA TURBINA HIDROCINÉTICA DE EE HORIZONTAL DISEÑADA. 


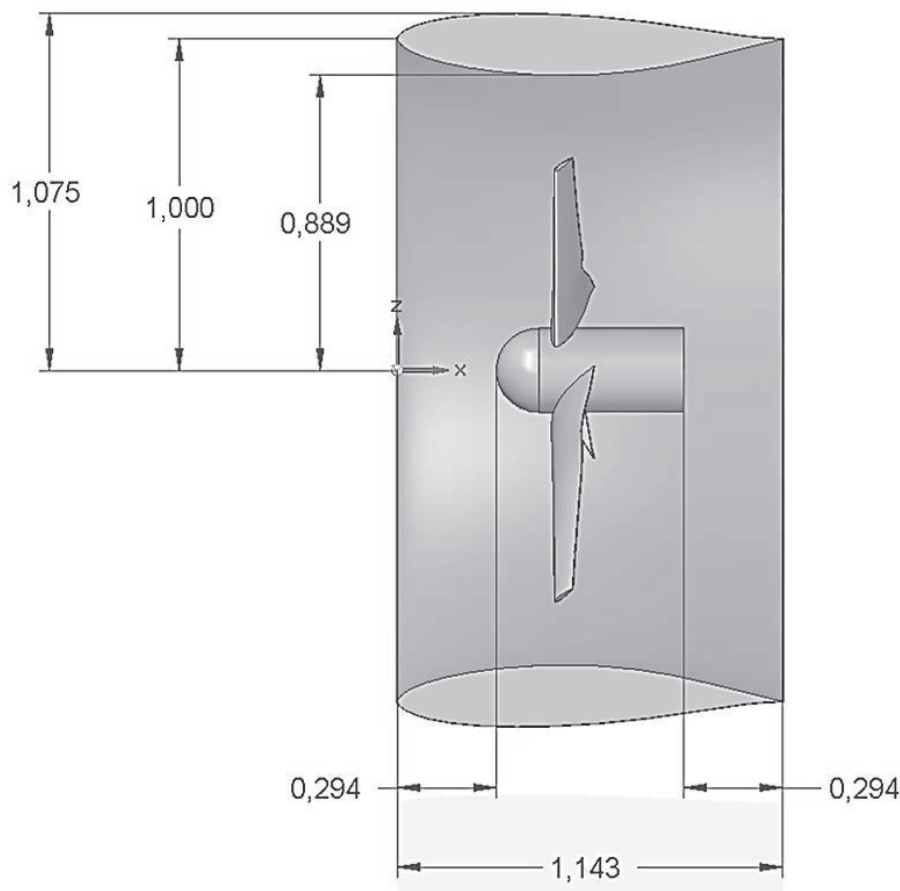

FIGURA 2. ESQUEMA DEL DIFUSOR ELABORADO.

Posteriormente, se generó el volumen circundante de la turbina hidrocinética con y sin difusor, el cual está compuesto por un volumen rotativo y otro estacionario (Fig. 3). El volumen rotativo es correspondiente al fluido sobre el cual se presenta una incidencia directa por la rotación del rotor. El cual fue utilizado para simular el movimiento de giro en dicha zona, y es representado con el color morado oscuro en la Fig. 3. Este fluido, presenta variaciones de velocidad a medida que el agua pasa a través de la turbina y se aleja de la misma. El fluido estacionario por su parte, representa el fluido del entorno en el cual se encuentra sumergido el modelo, que puede ser un río, mar, canal o entre otros. Este fluido es simbolizado por la zona transparente, verde y roja de la Fig. 3 .

La Fig. 3 presenta el volumen de fluido tridimensional de la turbina hidrocinética de eje horizontal y algunas de las dimensiones más representativas del mismo. La zona de color verde y color rojo son la entrada y salida del modelo, respectivamente. Las paredes del cubo y los álabes de la 
turbina hidrocinética, de color azul y la región de color morado claro que representa las paredes del difusor, fueron configuradas como paredes en la simulación. Tanto la salida como la pared externa del volumen estacionario, fueron configuradas como una apertura, para que el fluido pudiera tanto entrar como salir en dichas zonas. Por último, las áreas a la entrada y salida del difusor, simbolizadas con el color morado más oscuro, fueron establecidas como rotor congelado. Esto último debido a que la velocidad de giro de la turbina solamente se verá afectada por la velocidad de entrada del fluido.

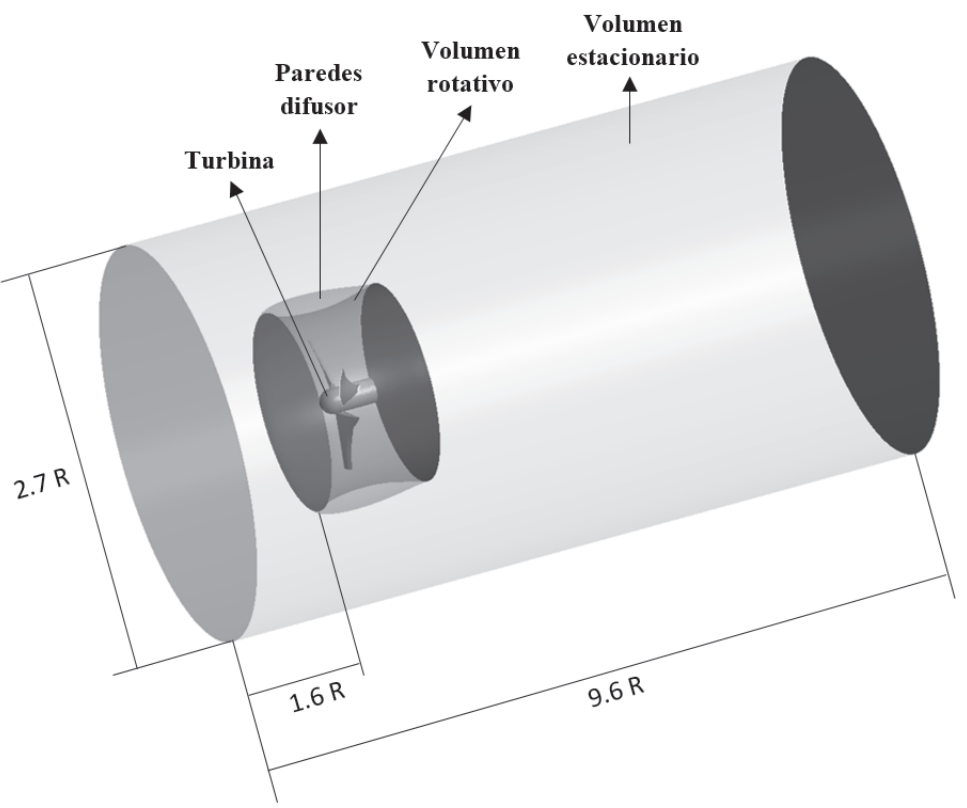

FIGURA 3. VOLUMEN DE FLUIDO DEL MODELO TRIDIMENSIONAL DE LA TURBINA HIDROCINÉTICA DE EJE HORIZONTAL Y SUS RESPECTIVAS DIMENSIONES EN FUNCIÓN DEL RADIO (R) DE LA TURBINA. 


\subsection{Discretización del modelo}

Ambos modelos fueron exportados al programa ANSYS V $16.2^{\circledR}$, donde se realizó el proceso de discretización. Se empleó una malla tetraédrica, con relevancia de +100 para una mayor precisión de los resultados. Este parámetro de relevancia permite controlar la finura de la malla para todo el modelo, con valores desde -100 (malla gruesa) hasta +100 (malla fina), proporcionando este último mayor precisión en los cálculos [9]. Se utilizó un tamaño mínimo de elementos de $1 \mathrm{~mm}$ en el borde de salida de los álabes, y un algoritmo de proximidad y curvatura. Se obtuvo una malla compuesta por $1.019 \mathrm{E}+7 \mathrm{y}$ $2.096 \mathrm{E}+7$ elementos, con $1.765 \mathrm{E}+6$ y $3.668 \mathrm{E}+6$ nodos, para la turbina con $\mathrm{y}$ sin difusor, respectivamente. Para ambos casos se obtuvieron métricas aceptables con valores máximos de oblicuidad inferiores a 0.87 , calidad ortogonal superiores a 0.98 y de relación de aspecto por debajo de 30.

La cantidad de elementos y nodos de malla para cada modelo, se estableció luego de realizar un estudio de convergencia de malla, a fin de garantizar la independencia de la misma con relación a los resultados arrojados. Se aseguró que el porcentaje de error fuera inferior al 4 \%. La Figura 4 y Figura 5 muestran el análisis de convergencia de malla para la turbina con y sin difusor, respectivamente. Para el análisis se utilizó el porcentaje de error relativo del torque generado por la turbina en función del número de nodos de malla. Para el análisis se emplearon 23 y 30 mallas diferentes con un rango de cantidad de nodos entre $1.108 \mathrm{E}+6$ y $6.974 \mathrm{E}+6$, y $2.07 \mathrm{E}+5$ y $1.336 \mathrm{E}+7$, para el modelo con y sin difusor, respectivamente. Ambas figuras presentan un pico cuando se utiliza una baja cantidad de nodos, el cual se debe a que la malla de dichos picos corresponde a la discretización de los modelos con la menor relevancia posible (-100). Esto se debe a que la cantidad de nodos y elementos de malla no son directamente proporcionales al incremento de la relevancia. El análisis de convergencia permitió determinar que los resultados se estabilizan a partir de los $1.7 \mathrm{E}+6$ y $3.3 \mathrm{E}+6$ nodos, garantizando una independencia de malla con relación a los resultados arrojados a partir de dichos valores. 


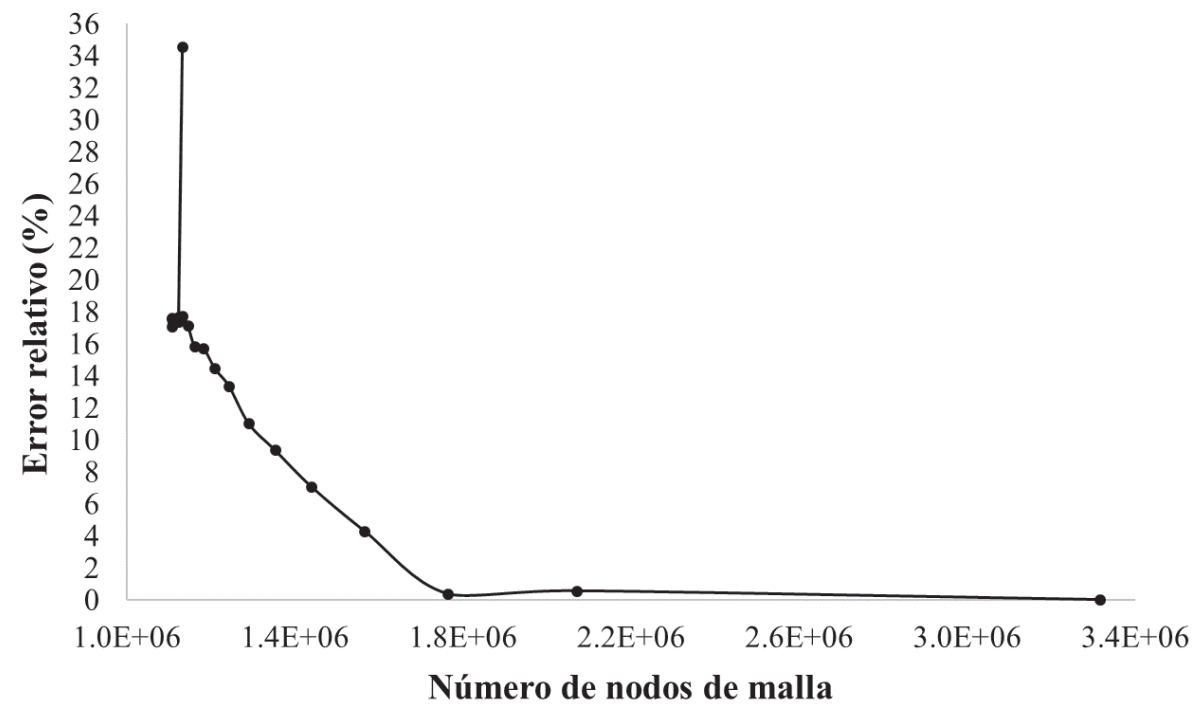

FIGURA 4. ANÁLISIS DE CONVERGENCIA PARA EL MODELO TRIDIMENSIONAL DE LA TURBINA HIDROCINÉTICA DE EJE HORIZONTAL CON DIFUSOR.

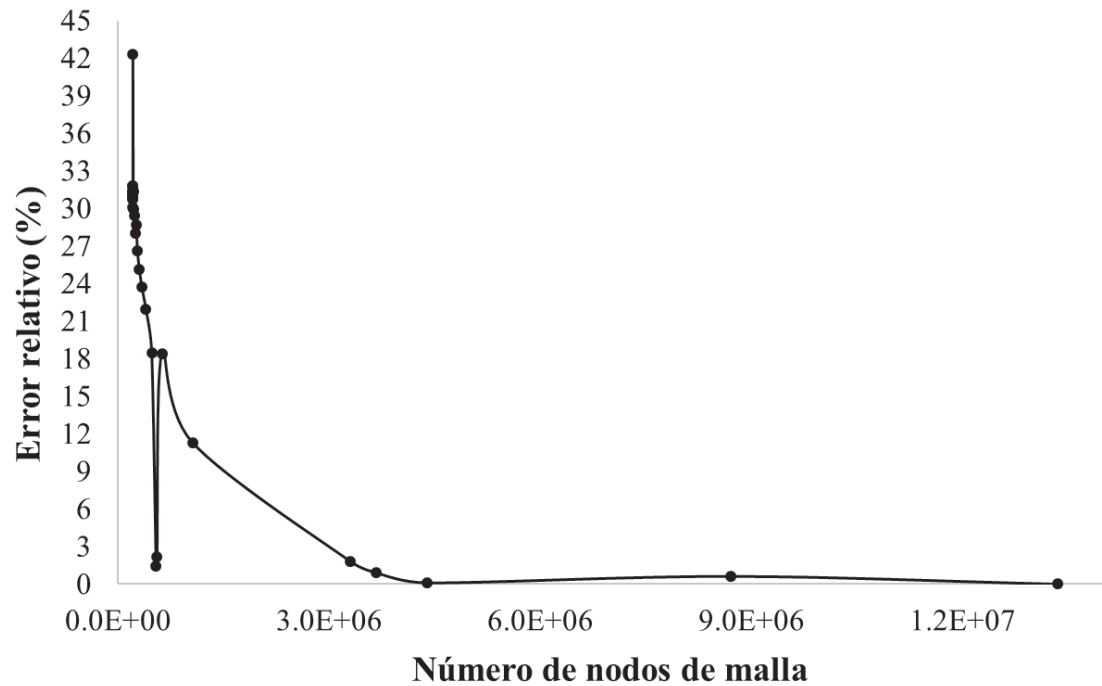

FIGURA 5. ANÁLISIS DE CONVERGENCIA PARA EL MODELO TRIDIMENSIONAL DE LA TURBINA HIDROCINÉTICA SIN DIFUSOR. 
Posteriormente, se hizo un análisis fluido-dinámico computacional (CFD) en el módulo CFX, utilizando una simulación transitoria por $6 \mathrm{~s}$ y $4 \mathrm{~s}$ para la turbina con y sin, respectivamente. El tiempo de simulación varió de un modelo a otro debido a que se llevó un constante chequeo de la variación del torque generado por la turbina respecto al tiempo para garantizar su estabilización. Se usó un paso de tiempo de $0.01 \mathrm{~s}$, un modelo de turbulencia k- $\varepsilon$ y se consideró agua a temperatura ambiente como fluido de trabajo. Como parámetros de entrada se empleó una velocidad del fluido de $1.5 \mathrm{~m} / \mathrm{s}$ y una variación de la velocidad angular con incrementos de 10 RPM, para un rango de o a 300 RPM. Una vez finalizada la simulación, se emplea la expresión matemática de torque (torque_ejerotación()@locación) para calcular su valor sobre los álabes de la turbina, por ser el área de interés. Esta expresión está contenida en la sección de resultados del módulo CFX de ANSYS ${ }^{\circledR}$. Con lo cual, se calculó la potencia generada por la turbina, al multiplicar el torque por la velocidad angular de la misma.

\section{Resultados}

En la Fig. 4 se presenta la potencia generada (W) por la turbina hidrocinética de eje horizontal sin y con difusor representadas con línea discontinua y continua, respectivamente, en función de la variación de la velocidad angular de 0 a 300 RPM.

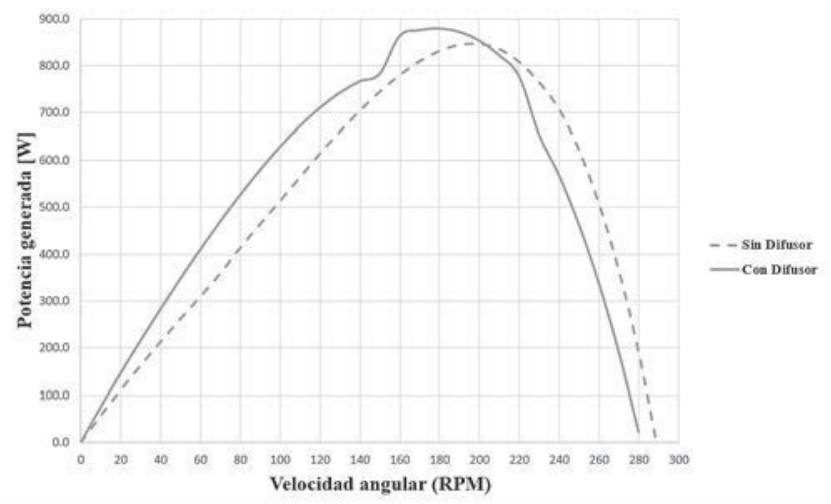

Figura 6. POTENCIA GENERADA (W) EN FUNCIÓN DE LA VELOCIDAD ANGULAR (RPM) POR LA TURBINA HIDROCINÉTICA SIN Y CON DIFUSOR. 
De la Fig. 4 se puede apreciar que la turbina con difusor (línea continua) alcanza una potencia máxima generada de $879.7 \mathrm{~W}$ cuando gira a 180 RPM, mientras que la turbina sin difusor (línea discontinua) alcanza $845.9 \mathrm{~W}$ a 200 RPM; representando un incremento de $3.84 \%$ de la potencia generada como respuesta a la influencia causada por la implementación del difusor. Esta influencia se ve reflejada a lo largo de las curvas de potencia, ya que al utilizar el difusor se genera una mayor potencia con velocidades angulares inferiores a las requeridas por la turbina sin difusor.

Otro aspecto a resaltar de la Fig. 4 es el hecho de que la turbina hidrocinética con difusor alcanza una potencia de generación de 854.1 W a 200 RPM, lo cual sigue estando por encima del punto de mayor generación de la turbina sin difusor a dicha velocidad de giro.

Los resultados de la turbina con difusor presentan una perturbación entre 150 y 160 RPM, lo cual genera una ligera modificación en el comportamiento que venía presentando la curva de potencia. Este fenómeno deberá ser estudiado con mayor detalle, a fin de entender sus causas y repercusiones.

\section{Conclusiones}

La implementación del difusor en la turbina hidrocinética de eje horizontal generó un incremento del 3.84 \% con relación a la turbina sin dicho elemento, lo cual podría ser considerado como un resultado poco significativo para el trabajo que podría traer consigo el desarrollo de este tipo de difusor, dada la complejidad de la curvatura presentada por el perfil hidrodinámico. Además, se presenta una gran diferencia al compararse dicho incremento con los resultados obtenidos por Gaden y Bibeau (2010), y Shahsavarifard et al. (2013) ${ }^{[6], ~[7] . ~ D o n d e ~ p o r ~ u t i l i z a r ~ u n ~ d i f u s o r ~ l o g r a r o n ~ a l c a n z a r ~ a u m e n t o s ~}$ en la potencia generada por la turbina de 3.1 veces y $91 \%$, respectivamente, con respecto a la turbina sin difusor.

Pese a lo anterior, un paso a seguir puede ser el análisis del mismo difusor, bajo diferentes posiciones de la turbina con respecto a la entrada del mismo e incluso evaluarlo a diferentes escalas, con el fin de mejorar la potencia de salida. 


\section{Referencias}

[1] F. Behrouzi, a Maimun, and M. Nakisa, "Review of Various Designs and Development in Hydropower Turbines," Int. J. Mech. Aerospace, Ind. Mechatronics Eng., vol. 8, no. 2, pp. 87-91, 2014.

[2] W. M. J. Batten, a. S. Bahaj, a. F. Molland, and J. R. Chaplin, "The prediction of the hydrodynamic performance of marine current turbines," Renew. Energy, vol. 33, no. 5, pp. 1085-1096, 2008.

[3] a. S. Bahaj, W. M. J. Batten, and G. McCann, "Experimental verifications of numerical predictions for the hydrodynamic performance of horizontal axis marine current turbines," Renew. Energy, vol. 32, no. 15, pp. 2479-2490, 2007.

[4] L. Chen, F. L. Ponta, and L. I. Lago, "Perspectives on innovative concepts in windpower generation,” Energy Sustain. Dev., vol. 15, no. 4, pp. 398-410, 2011.

[5] T. Y. Chen, Y. T. Liao, and C. C. Cheng, "Development of small wind turbines for moving vehicles: Effects of flanged diffusers on rotor performance," Exp. Therm. Fluid Sci., vol. 42, pp. 136-142, 2012.

[6] D. L. F. Gaden and E. L. Bibeau, "A numerical investigation into the effect of diffusers on the performance of hydro kinetic turbines using a validated momentum source turbine model," Renew. Energy, vol. 35, no. 6, pp. 1152-1158, Jun. 2010.

[7] M. Shahsavarifard, E. L. Bibeau, and V. Chatoorgoon, "Effect of shroud on the performance of horizontal axis hydrokinetic turbines," Ocean Eng., vol. 96, pp. 215-225, Mar. 2015.

[8] E. Chica, F. Perez, A. Rubio-Clemente, and S. Agudelo, "Design of a hydrokinetic turbine," WIT Trans. Ecol. Environ., vol. 195, pp. 137-148, 2015.

[9] ANSYS, "Relevance." 2016. 